\title{
A split- and delay-unit for the European XFEL
}

\author{
Sebastian Roling $^{1 *}$, Stefan Braun ${ }^{2}$, Peter Gawlitza ${ }^{2}$, Liubov Samoylova $^{3}$, Björn Siemer ${ }^{1}$, \\ Harald Sinn $^{3}$, Frank Siewert ${ }^{4}$, Frank Wahlert ${ }^{1}$, Michael Wöstmann ${ }^{1}$ and Helmut Zacharias ${ }^{1 *}$ \\ ${ }^{1}$ Physikalisches Institut, WWU Münster, Wilhelm-Klemm Straße 10, 48149 Münster, Germany \\ ${ }^{2}$ Fraunhofer IWS Dresden, Winterbergstraße 28, 01277 Dresden, Germany \\ ${ }^{3}$ European XFEL GmbH, Albert-Einstein-Ring 19, 22761 Hamburg, Germany \\ ${ }^{4}$ HZB, Albert-Einstein-Straße 15,12489 Berlin, Germany \\ *corresponding authors: s_roli02@uni-muenster.de, h.zacharias@uni-muenster.de
}

\begin{abstract}
For the European XFEL [1] an X-ray split- and delay-unit (SDU) is built covering photon energies from $5 \mathrm{keV}$ up to $20 \mathrm{keV}$ [2]. This SDU will enable time-resolved x-ray pump / $\mathrm{x}$-ray probe experiments as well as sequential diffractive imaging [3] on a femtosecond to picosecond time scale. Further, direct measurements of the temporal coherence properties will be possible by making use of a linear autocorrelation. The set-up is based on geometric wavefront beam splitting, which has successfully been implemented at an autocorrelator at FLASH [4]. The X-ray FEL pulses will be split by a sharp edge of a silicon mirror coated with $\mathrm{Mo} / \mathrm{B}_{4} \mathrm{C}$ multi layers. Both partial beams will then pass variable delay lines. For different wavelengths the angle of incidence onto the multilayer mirrors will be adjusted in order to match the Bragg condition. For a photon energy of $h v=20 \mathrm{keV}$ a grazing angle of $\theta=0.57^{\circ}$ has to be set, which results in a footprint of the beam $(6 \sigma)$ on the mirror of $1=120 \mathrm{~mm}$. At this photon energy the reflectance of a Mo/ $\mathrm{B}_{4} \mathrm{C}$ multi layer coating with a multi layer period of $d=3.2 \mathrm{~nm}$ and $\mathrm{N}=200$ layers amounts to $\mathrm{R}=0.92$. In order to enhance the maximum transmission for photon energies of $h v=8 \mathrm{keV}$ and below, a Ni/ $\mathrm{B}_{4} \mathrm{C}$ multilayer coating can be applied beside the $\mathrm{Mo} / \mathrm{B}_{4} \mathrm{C}$ coating for this spectral region. Because of the different incidence angles, the path lengths of the beams will differ as a function of wavelength. Hence, maximum delays between $+/-2.5 \mathrm{ps}$ at $\mathrm{h} v=20 \mathrm{keV}$ and up to $+/-23 \mathrm{ps}$ at $\mathrm{h} v=5 \mathrm{keV}$ will be possible.
\end{abstract}

\section{INTRODUCTION}

The emergence of new hard x-ray sources providing ultrashort and ultrabright light pulses allows for new classes of $\mathrm{x}$ ray experiments. This is a great challenge for optical instrumentation. In addition to the already operating LCLS at the Stanford Linear Accelerator Center (USA) [5] and SACLA in Japan [6] the European XFEL is now under construction in Hamburg (Germany). Operating at electron bunch energies of $17.5 \mathrm{GeV}$ the machine will provide photon energies between $\mathrm{h} v=3 \mathrm{keV}$ and $\mathrm{h} v=24 \mathrm{keV}$ at the undulator sources SASE1 and 2. Pulse energies of presumably $\mathrm{E}_{\text {pulse }}=2 \mathrm{~mJ}$

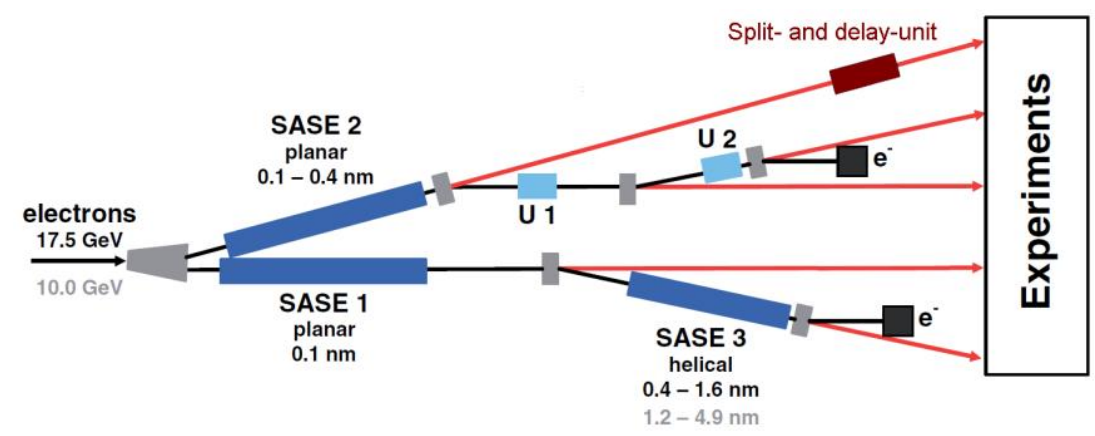

Fig. 1: A possible point of integration of the SDU into the beam line SASE2 $130 \mathrm{~m}$ in front of the experimental hall, after [1].

Advances in X-ray Free-Electron Lasers II: Instrumentation, edited by

Thomas Tschentscher, Kai Tiedtke, Proc. of SPIE Vol. 8778, 87781G · @ 2013

SPIE $\cdot$ CCC code: $0277-786 \mathrm{X} / 13 / \$ 18 \cdot$ doi: $10.1117 / 12.2027029$ 
and a pulse duration on the order of $\tau_{\text {pulse }}=100 \mathrm{fs}$ [1] are expected. In the burst mode very high repetition rates of 2700 pulses at $4.5 \mathrm{MHz}$ per burst at a repetition rate of $10 \mathrm{~Hz}$ are possible, due to superconducting accelerators. In order to gain information about the temporal properties of the x-ray pulses, like temporal coherence and pulse-duration, two jitter-free pulse replicas are required. Also for x-ray pump / x-ray probe experiments and for time-resolved diffractive imaging a split- und delay unit is needed.

Optical split- and delay-units or autocorrelators for the infrared, visible and ultraviolet spectral regime based on a Michelson or a Mach-Zehnder set-up are in general use, for example for the measurement of pulse durations of femtosecond lasers. Recently, a compact x-ray split- and delay-unit that utilizes crystal optics has been developed [7]. Due to the $90^{\circ}$ geometry a compact and sturdy design was possible and a large delay of $\Delta \mathrm{t}=2.6 \mathrm{~ns}$ was achieved with this device. A potential drawback of crystal optics is their spectral selectivity. For example $\mathrm{GaAs}(400)$ or $\mathrm{Si}(111)$ yield a spectral bandwidth of $\Delta \mathrm{E}=0.5 \mathrm{eV}$ for $\mathrm{Cu} \mathrm{K}_{\alpha}$ radiation. In comparison, the SASE1/2 undulators of the European XFEL will have a spectral bandwidth in the order of $0.08 \%-0.2 \%$, which corresponds to $\Delta \mathrm{E} \sim 8 \mathrm{eV}$ at $\mathrm{h} v=8 \mathrm{keV}$. On the one hand, the temporal coherence of the FEL pulses is enhanced due to the narrow bandwidth. On the other hand, a measurement of the original coherence properties is not possible. Unfortunately, the special diffraction spots that have to be used allow a transmission of only $\mathrm{T} \sim 0.6 \%$ at $\mathrm{h} v=8.36 \mathrm{eV}$.

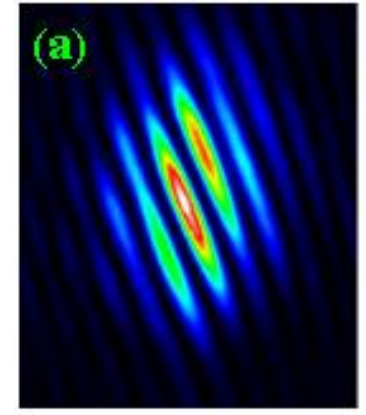

Fig.2: Interferences of FLASH at zero delay (a) and at $\Delta \mathrm{t}=50 \mathrm{fs}$; $\lambda=24 \mathrm{~nm}$.

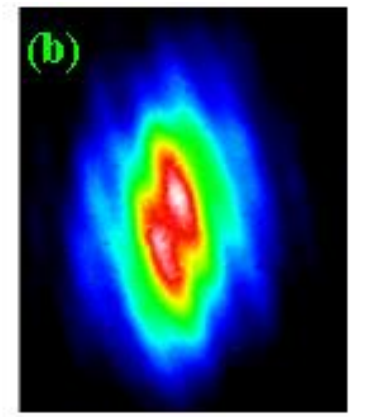

(1)
$y=$ temporal coherence properties $[4,8]$ as well as the pulse duration [9] of a soft x-ray FEL have successfully been measured for the first time. Fig. 2 exemplarily shows interference fringes of a single FLASH pulse at $\lambda=24 \mathrm{~nm}$ at zero and at $50 \mathrm{fs}$ delay, respectively. The overlap of the two partial beams is $\Delta \mathrm{x} \approx 1.2 \mathrm{~mm}$ which corresponds to $\sim 44 \%$ of the beam diameter in this case where an $1 \mathrm{~mm}$ aperture is set $65 \mathrm{~m}$ in front of the detector at the center of the beam profile. In these particular cases the contrast of the interference fringes yield a visibility $\left(V=I_{\max }-I_{\min } / I_{\max }+I_{\min }\right.$, where $I_{\max }$ and $\mathrm{I}_{\min }$ are the maximum and minimum intensities.) of $\mathrm{V}=0.82$ and $\mathrm{V}=0.07$, respectively. Coherence times (FWHM) between $\tau_{\mathrm{c}}=6$ fs for $\lambda=32 \mathrm{~nm}$ and $\tau_{\mathrm{c}}=2.9 \mathrm{fs}$ for $\lambda=8 \mathrm{~nm}$ were measured [8].

Further, ionization dynamics in expanding clusters have been investigated by XUV pump / XUV probe spectroscopy [10] and femtosecond sequential imaging has been realized for the first time [3].

The new SDU at the European XFEL will enable similar experiments in the x-ray spectral regime. While for the energy range of FLASH carbon coated silicon mirrors still yield a sufficient reflectivity at photon energies up about to $\mathrm{h} v=200 \mathrm{eV}$, this will not be the case for the hard $\mathrm{x}$-ray pulses of the European XFEL. Therefore, Si-substrates coated with multilayers will be utilized. Furthermore, there is an option to use single metal layers (e.g. platinum) instead of multilayers, which would enable the SDU to be used for the soft $x$-ray beamline SASE 3.

\section{OPTICAL CONCEPT}

The high absorbance and the small reflectivity at large incident angles are severe limitations for optical instrumentation in the $\mathrm{x}$-ray range and therefore demand for a grazing incident geometry. The optical concept will have to meet various requirements, like: A high reflectivity, transmission of the whole spatial beam-profile, delay with sub-fs resolution, a large delay range, and the wide photon energy range of the XFEL $(5-20 \mathrm{keV})$. These properties have to be achieved with a minimal disturbance of the beam position and direction, a high mechanical stability making a temporal resolution 
in the sub 100 attosecond regime feasible. The design and construction should of course incorporate elements, which allow a realization of the SDU in a practicable size.

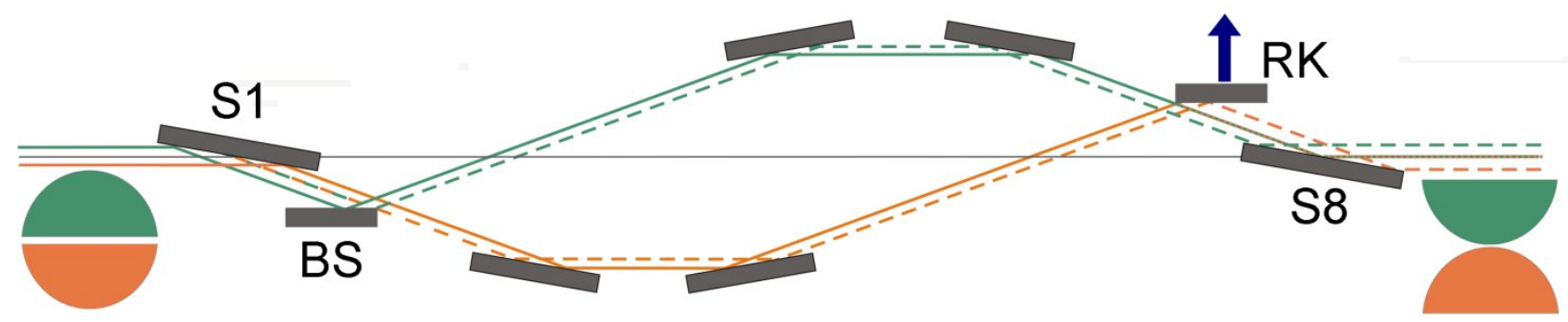

Fig.3: Schematic drawing of the optical layout of the x-ray split- and delay-unit.

In order to meet these requirements a point symmetric optical concept based on a geometrical wavefront beam splitter and multilayer Bragg coatings which permit larger grazing angles has been developed. The whole set-up of the optical pathway is schematically shown in Fig. 3. The XFEL beam enters the SDU from the left side and is reflected by the first mirror (S1) downwards in the direction of the beam splitter (BS). The lower green part of the beam is reflected into the upper delay arm while the upper orange part passes the sharp edge in the direction of the lower delay arm. The mirrors of both delay arms can be moved along the split beam direction in order to introduce a temporal delay between both partial beams. After the orange beam has passed the lower delay line it is reflected by the recombination mirror (RK) in the direction of the last mirror (S8). The green beam passes the sharp edge of the recombination mirror unaffected. Thus, in this point symmetric concept the recombination mirror acts as the counterpart of the beam splitter. The last mirror (S8) reflects both beams into their original direction. It should be noted that the beam shape of both arms is rotated by $180^{\circ}$ due to the odd number of reflections. In order to perform experiments the beams will have to be overlapped. This can be achieved by slightly rotating the recombination mirror, RK.

As already mentioned the mirrors are intended to work at grazing incidence angles. For photon energies from $\mathrm{h} v=5 \mathrm{keV}$ to $\mathrm{h} v=20 \mathrm{keV}$ multilayers will be used on the mirrors which possess high reflectivity. Since for multilayers the grazing angle depends on the wavelength, the mirrors have to be aligned for different wavelengths.

Table 1: The total transmission and the maximum delay times.

\begin{tabular}{|c|c|r|c|c|}
\hline $\begin{array}{c}\text { Photonenergy } \\
{[\mathrm{keV}]}\end{array}$ & $\begin{array}{c}\text { Multilayer } \\
\text { Top-Material }\end{array}$ & $\begin{array}{l}\text { Angle (BS) } \\
{\left[{ }^{\circ}\right]}\end{array}$ & $\begin{array}{c}\text { Delay } \\
{[\mathrm{ps}]}\end{array}$ & Transmission \\
\hline 4 & $\mathrm{Ni}$ & 4,58 & 36 & 0,16 \\
\hline 5 & $\mathrm{Ni}$ & $\mathbf{3 , 6 6}$ & 23 & $\mathbf{0 , 2 6}$ \\
\hline 6 & $\mathrm{Ni}$ & $\mathbf{3 , 0 7}$ & $\mathbf{1 6}$ & $\mathbf{0 , 3 5}$ \\
\hline 8 & $\mathrm{Ni}$ & $\mathbf{2 , 3 1}$ & 9 & $\mathbf{0 , 4 8}$ \\
\hline 10 & $\mathrm{Ni}$ & $\mathbf{1 , 8 3}$ & $\mathbf{6}$ & $\mathbf{0 , 2 1}$ \\
\hline $\mathbf{1 0}$ & $\mathrm{Mo}$ & $\mathbf{2 , 2 8}$ & $\mathbf{9}$ & $\mathbf{0 , 2 9}$ \\
\hline $\mathbf{1 2}$ & Mo & $\mathbf{1 , 9 0}$ & $\mathbf{6}$ & $\mathbf{0 , 3 5}$ \\
\hline $\mathbf{1 8}$ & Mo & $\mathbf{1 , 2 7}$ & $\mathbf{3}$ & $\mathbf{0 , 4 3}$ \\
\hline $\mathbf{2 0}$ & Mo & $\mathbf{1 , 1 4}$ & $\mathbf{2 . 5}$ & $\mathbf{0 , 2 3}$ \\
\hline
\end{tabular}

\section{MIRRORS}

\subsection{Multilayer coating}

The silicon mirror substrates will be coated with $\mathrm{Mo} / \mathrm{B}_{4} \mathrm{C}$ multilayers. Therefore, the grazing angle $\theta$ depends on the photon energy of the incident FEL beam. As it is obvious from Fig. 3 the grazing angle $\theta$ under which the beam splitter and the recombination mirror are to be positioned will be twice as large as the grazing angle of the other mirrors. Hence, different multilayer periods will have to be utilized for these two mirrors. In this regard, it has to be ensured that a 
Table 2: Angular width of the multilayer reflection curves.

\begin{tabular}{|c|c|c|c|c|c|c|}
\hline $\begin{array}{c}\text { photon energy } \\
{[\mathrm{keV}]}\end{array}$ & coating & $\begin{array}{c}\text { multilayer period } \\
{[\mathrm{nm}]}\end{array}$ & $\begin{array}{c}\text { gracing angle } \\
\theta\left[{ }^{\circ}\right]\end{array}$ & $\begin{array}{c}\text { angular width } \\
\Delta \theta \text { FWHM }[\mu \mathrm{rad}]\end{array}$ & $\begin{array}{c}\Delta \theta 90 \% \\
{[\mu \mathrm{rad}]}\end{array}$ & reflectivity \\
\hline 20 & $\mathrm{Mo} / \mathrm{B}_{4} \mathrm{C}$ & 3.20 & 0.57 & 146 & 119 & 0.92 \\
20 & $\mathrm{Mo} / \mathrm{B}_{4} \mathrm{C}$ & 1.57 & 1.14 & 111 & 58 & 0.49 \\
\hline 18 & $\mathrm{Mo} / \mathrm{B}_{4} \mathrm{C}$ & 3.20 & 0.63 & 246 & 191 & 0.94 \\
18 & $\mathrm{Mo} / \mathrm{B}_{4} \mathrm{C}$ & 1.57 & 1.27 & 158 & 75 & 0.72 \\
\hline 16 & $\mathrm{Mo} / \mathrm{B}_{4} \mathrm{C}$ & 3.20 & 0.71 & 263 & 214 & 0.93 \\
16 & $\mathrm{Mo} / \mathrm{B}_{4} \mathrm{C}$ & 1.57 & 1.42 & 172 & 96 & 0.72 \\
\hline 12 & $\mathrm{Mo} / \mathrm{B}_{4} \mathrm{C}$ & 3.20 & 0.95 & 351 & 258 & 0.89 \\
12 & $\mathrm{Mo} / \mathrm{B}_{4} \mathrm{C}$ & 1.57 & 1.90 & 228 & 105 & 0.69 \\
10 & $\mathrm{Ni} / \mathrm{B}_{4} \mathrm{C}$ & 4.00 & 0.92 & 419 & 286 & 0.73 \\
10 & $\mathrm{~W} / \mathrm{B}_{4} \mathrm{C}$ & 1.94 & 1.83 & 209 & 72 & 0.74 \\
\hline 8 & $\mathrm{Mo} / \mathrm{B}_{4} \mathrm{C}$ & 3.20 & 1.43 & 526 & 300 & 0.79 \\
8 & $\mathrm{Mo} / \mathrm{B}_{4} \mathrm{C}$ & 1.57 & 2.85 & 333 & 156 & 0.61 \\
8 & $\mathrm{Ni} / \mathrm{B}_{4} \mathrm{C}$ & 4.00 & 1.15 & 575 & 491 & 0.92 \\
8 & $\mathrm{~W} / \mathrm{B}_{4} \mathrm{C}$ & 1.94 & 2.31 & 381 & 246 & 0.68 \\
\hline 6 & $\mathrm{Mo} / \mathrm{B}_{4} \mathrm{C}$ & 3.20 & 1.90 & 772 & 344 & 0.69 \\
6 & $\mathrm{Mo} / \mathrm{B}_{4} \mathrm{C}$ & 1.57 & 3.80 & 456 & 189 & 0.52 \\
6 & $\mathrm{Ni} / \mathrm{B}_{4} \mathrm{C}$ & 4.00 & 1.54 & 842 & 561 & 0.89 \\
6 & $\mathrm{~W} / \mathrm{B}_{4} \mathrm{C}$ & 1.94 & 3.07 & 509 & 333 & 0.57 \\
\hline 5 & $\mathrm{Mo} / \mathrm{B}_{4} \mathrm{C}$ & 3.20 & 2.28 & 842 & 344 & 0.60 \\
5 & $\mathrm{Mo} / \mathrm{B}_{4} \mathrm{C}$ & 1.57 & 4.56 & 526 & 189 & 0.45 \\
5 & $\mathrm{Ni} / \mathrm{B}_{4} \mathrm{C}$ & 4.00 & 1.85 & 1000 & 649 & 0.86 \\
5 & $\mathrm{~W} / \mathrm{B}_{4} \mathrm{C}$ & 1.94 & 3.66 & 614 & 333 & 0.48 \\
\hline
\end{tabular}

maximum total transmission over the whole photon energy range is achieved. A promising combination of a multilayer periodicity of $\mathrm{d}=3.2 \mathrm{~nm}$ for the delay mirrors and one of $\mathrm{d}=1.57 \mathrm{~nm}$ for the beam splitter and recombination mirrors is shown in Fig. 4. It is clearly obvious that for photon energies of $h v=8 \mathrm{keV}$ and lower the reflectivity of the Mo/ $\mathrm{B}_{4} \mathrm{C}$ multilayers significantly decreases.
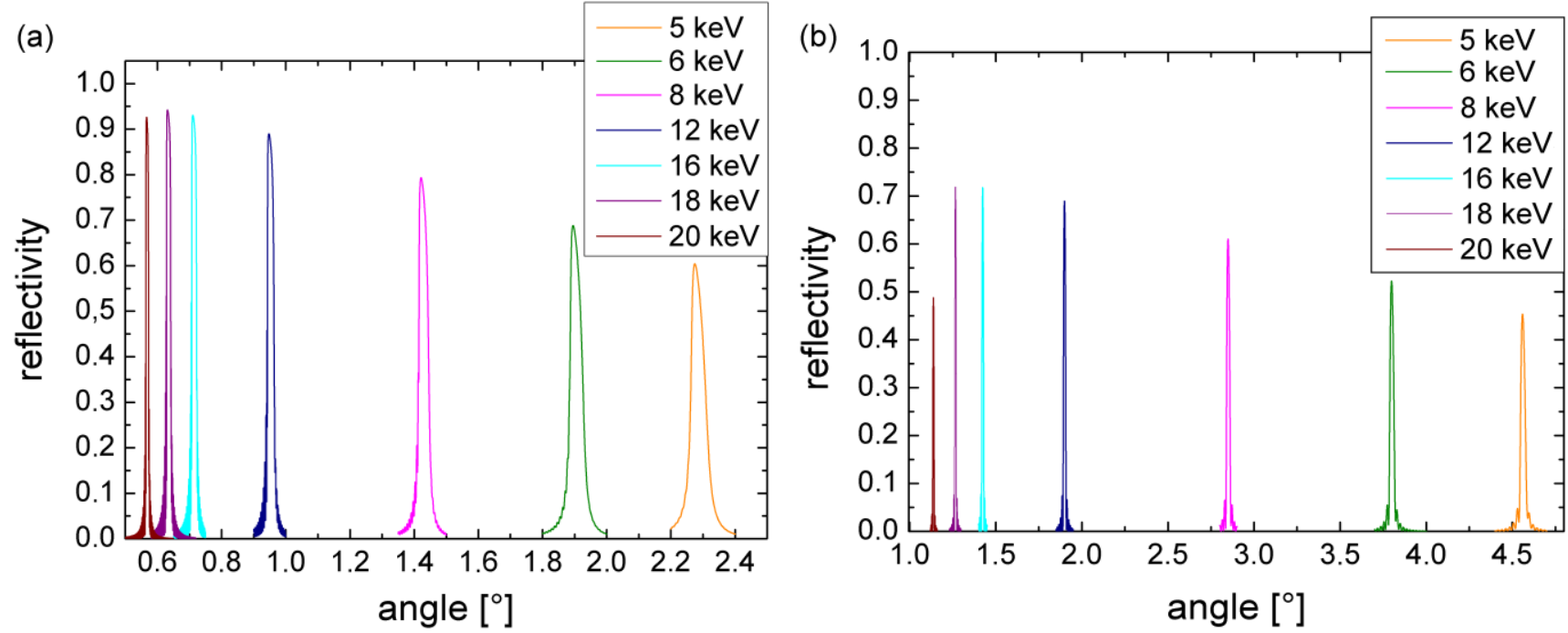

Fig. 4: Simulation of the reflectivity of $\mathrm{Mo} / \mathrm{B}_{4} \mathrm{C}$ multilayer coatings with a period $\mathrm{d}=3.2 \mathrm{~nm}$ for the delay mirrors $(\mathrm{a})$ and $\mathrm{d}=1.57$ $\mathrm{nm}$ for the beamsplitter and recombination mirror (b). 
Since the mirror substrates have a width of $25 \mathrm{~mm}$ and the beam diameter at the position of the SDU is smaller than 9.7 $\mathrm{mm}(6 \sigma$ at $\mathrm{h} v=5 \mathrm{keV})$ different multilayer coatings can be applied beside each other. In order to enhance the transmission for this spectral region a different coating with $\mathrm{Ni} / \mathrm{B}_{4} \mathrm{C}$ for the delay-mirrors and $\mathrm{W} / \mathrm{B}_{4} \mathrm{C}$ for the beamsplitter and recombination mirror can be applied beside the $\mathrm{Mo} / \mathrm{B}_{4} \mathrm{C}$ coating.The reflectivity for such a combination of coatings with a multilayer period of $d=1.94 \mathrm{~nm}\left(\mathrm{~W} / \mathrm{B}_{4} \mathrm{C}\right)$ for the beam splitter and recombination mirrors and $\mathrm{d}=4 \mathrm{~nm}$ $\left(\mathrm{Ni} / \mathrm{B}_{4} \mathrm{C}\right)$ for the other mirrors is given in Table 2 .

Table 2 also shows the angular width $\Delta \theta$ of the reflection curves (FWHM and $90 \%$ of the maximum reflectivity). These values are of utmost importance because as it was mentioned before, the recombination mirror has to be slightly turned in order to achieve a full spatial overlap of the two half beams in the experimental hall. Depending on the different beam sizes for different photon energies these angles vary between $\alpha=13.4 \mu \mathrm{rad}$ at $\mathrm{h} \nu=20 \mathrm{keV}$ and $\alpha=38 \mu \mathrm{rad}$ at $\mathrm{h} v=5 \mathrm{keV}$, if the SDU is integrated into the beamline $130 \mathrm{~m}$ in front of the experimental hall. This means that even for the narrowest reflection curve (beamsplitter at $\mathrm{h} v=20 \mathrm{keV}, \Delta \theta=58 \mu \mathrm{rad}$ ) the criterion for a full overlap of both half beams is fulfilled more than four fold.

\subsection{Mirror substrates}

An inspection of the mirror substrates is essential to check the optics quality. In addition such measurements allow to characterize the state of the optics under different clamping conditions. A typical T-shaped mirror $(1=380 \mathrm{~mm})$ as proposed for the SDU was inspected under three different supporting conditions in the face-up configuration:

1. the mirror was supported by a cloth on a ultra-flat Zerodur substrate $(\mathrm{R}>1000 \mathrm{~km})$.

2. the mirror was supported by gauge pieces located at the Bessel positions

3. the mirror was supported by gauge pieces located $25 \mathrm{~mm}$ from the mirror edges

The measurements were performed by means of the BESSY-NOM slope measuring profiler [11]. The mirror was measured along the centre line in meridional direction. The sampling spacing was $0.2 \mathrm{~mm}$.
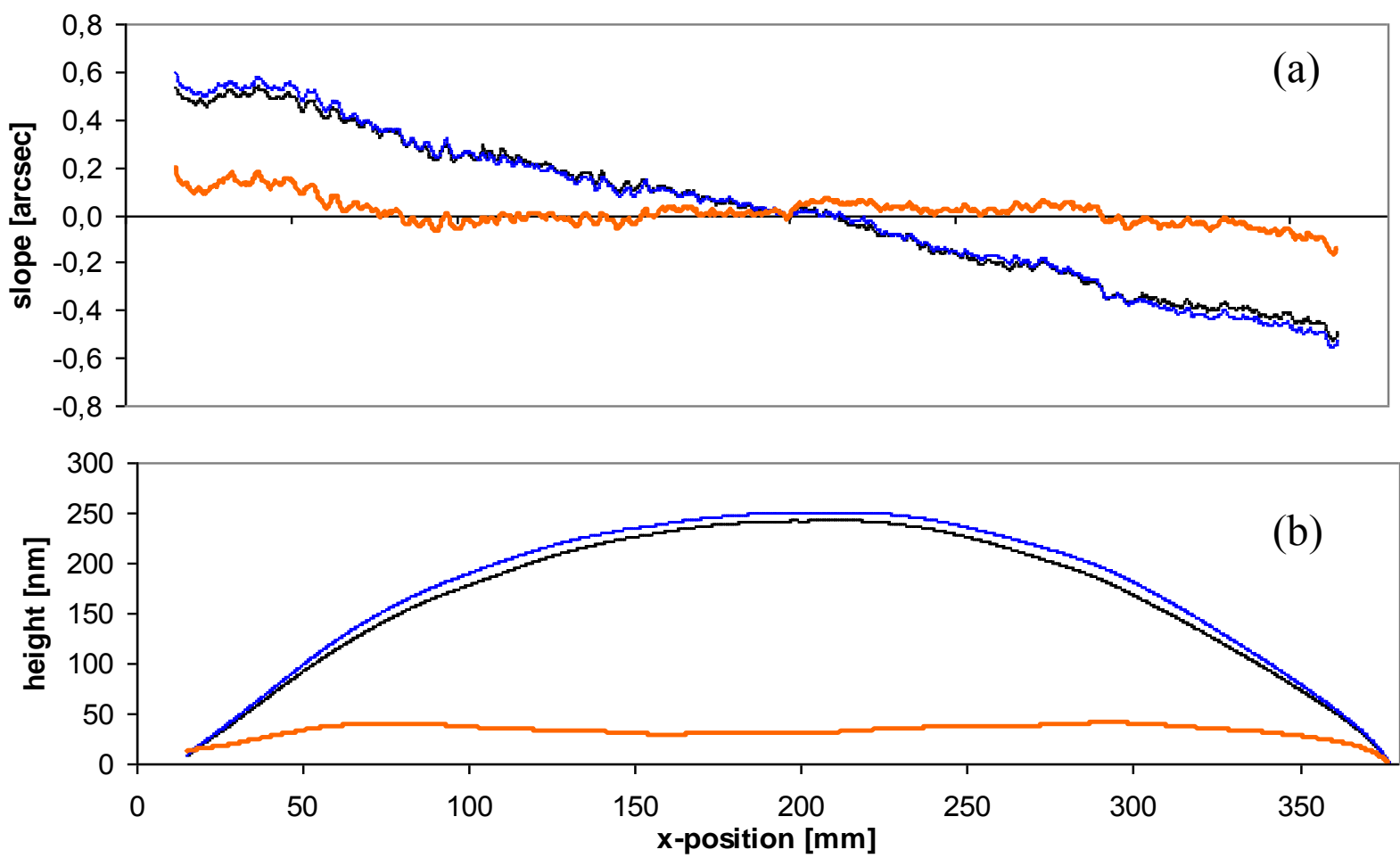

Fig. 5: Profiles of slope (a) and corresponding height (b) at centre line of the beam splitter mirror. The mirror is supported by a cloth on a ultra-flat Zerodur substrate (black), by gauge pieces at the Bessel positions (blue) and by gauge pieces $25 \mathrm{~mm}$ from the edges (orange) 
The spatial resolution covers a frequency range from $1.5 \mathrm{~mm}$ to aperture length (this is limited by the autocollimator aperture size of $2 \mathrm{~mm}$ applied for the measurements). Fig. 5 shows the slope and height profiles (absolute data) for the three different configurations.

The flat surface (configuration 1) as well as the Bessel mounting (2) show a curvature characterized by a slope of $>0.3 \mathrm{arcsec}(\mathrm{rms})$ and radii of curvature of $<100 \mathrm{~km}$. Placing the mirror on gauge pieces $25 \mathrm{~mm}$ from the edges (3) gives a demonstration for an option how the substrate can be tuned to a three times improved performance. For this case the slope is $<0.1 \operatorname{arcsec}(\mathrm{rms})$ and a radius of curvature of $>400 \mathrm{~km}$ is found. The peak-to-valley figure deviation is improved to about $40 \mathrm{~nm}$ peak-to-valley compared to $240-250 \mathrm{~nm}$ peak-to-valley initially. Figure 6 shows a comparison of state (2) and (3) by performing a slope mapping with the NOM.

\section{SLOPE DISTRIBIUTION IN MERIDIONAL DIRECTION}

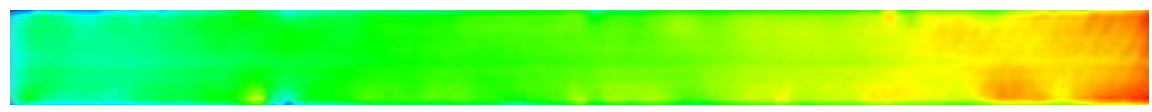

(2) essel- osition
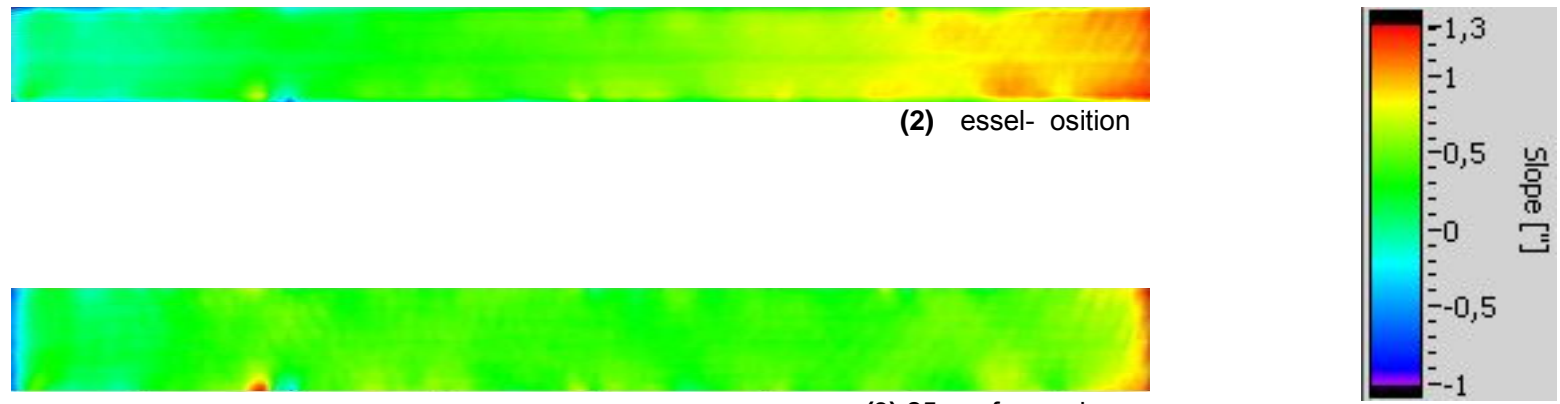

(3) $25 \mathrm{~mm}$ from ed es

MIRROR TOPOGRAPHY ACCORDING MERIDIONAL

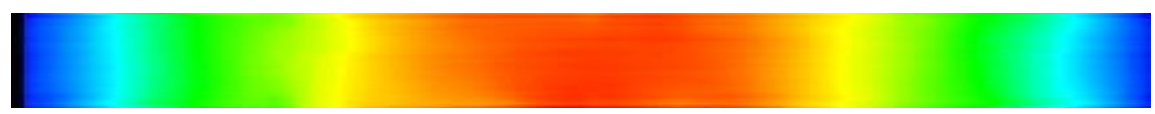

(2) essel- osition
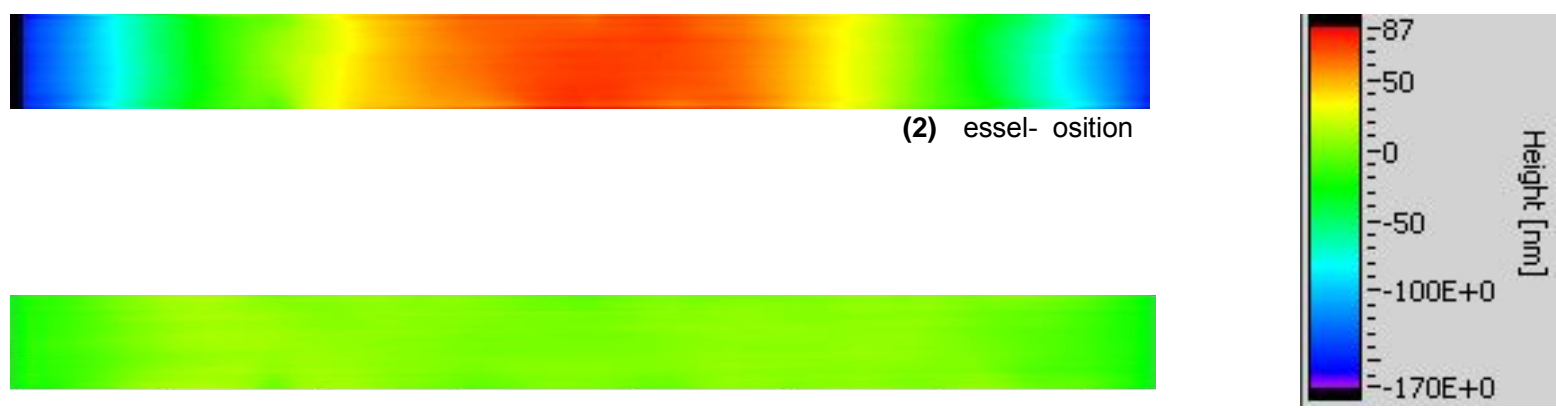

(3) $25 \mathrm{~mm}$ from ed es

Fig.6: Comparison of state (2) and (3) in terms of meridional slope mapping and height data achieved by integration of the slope mapping data

\subsection{Simulation of diffraction effects}

Since the European XFEL will provide the experiments with coherent radiation diffraction effects from the non-ideal mirror surfaces and especially from the sharp edge where the beam is split into two partial beams have to be evaluated. For the simulation shown in Fig.7 the surface profile of the beam splitter supported $25 \mathrm{~mm}$ from the edges (orange line in Fig.5) was taken. The simulation was made for a position of the SDU of $100 \mathrm{~m}$ in front of the experimental hall and a photon energy of $h v=5 \mathrm{keV}$, which represents a typical photon energy where the SDU will operate at SASE1 or SASE2. An idealized sharp edge of the mirror was assumed. In Fig. 7(a) the simulation of the partial beam that passes the beam splitter without being reflected is shown (orange beam in Fig. 3). Figure 7(b) shows vertical cut at $\mathrm{x}=0 \mathrm{~mm}$. The other partial beam that is reflected (green beam in Fig. 3) looks similar, but flipped horizontally. The fringes caused by the diffraction at the sharp edge are clearly obvious. The vertical modulations result from the offset mirrors of the beamline. They are caused by two factors: First, for a given angle of incidence, the beamline mirrors are to short to accept the full footprint of the XFEL, which leads to diffraction. Second, height errors of the mirror surface ( $2 \mathrm{~nm}$ peak 
to valley) disturb the wave front. Since the beamline mirrors do not cut the XFEL beam in the middle, where the intensity reaches its maximum, the visibility of the vertical fringes $(\mathrm{V}=0.09)$ is not as high as it is for the horizontal fringes $(\mathrm{V}=0.33)$, that are caused by the beam splitter of the SDU. In comparison, the fringes caused by coherent twobeam interference reach values of $\mathrm{V}=0.8$ and higher if the delay between the partial beams is zero, see also Fig. 2 .

Recently a new scheme has been developed, using Be-lenses in order to slightly collimate the XFEL beam. With this the footprint of the beam on the mirrors of the SDU will be about three times shorter than originally assumed. Therefore shorter substrates $(1=190 \mathrm{~mm})$ with a better quality of the surface will be foreseen.
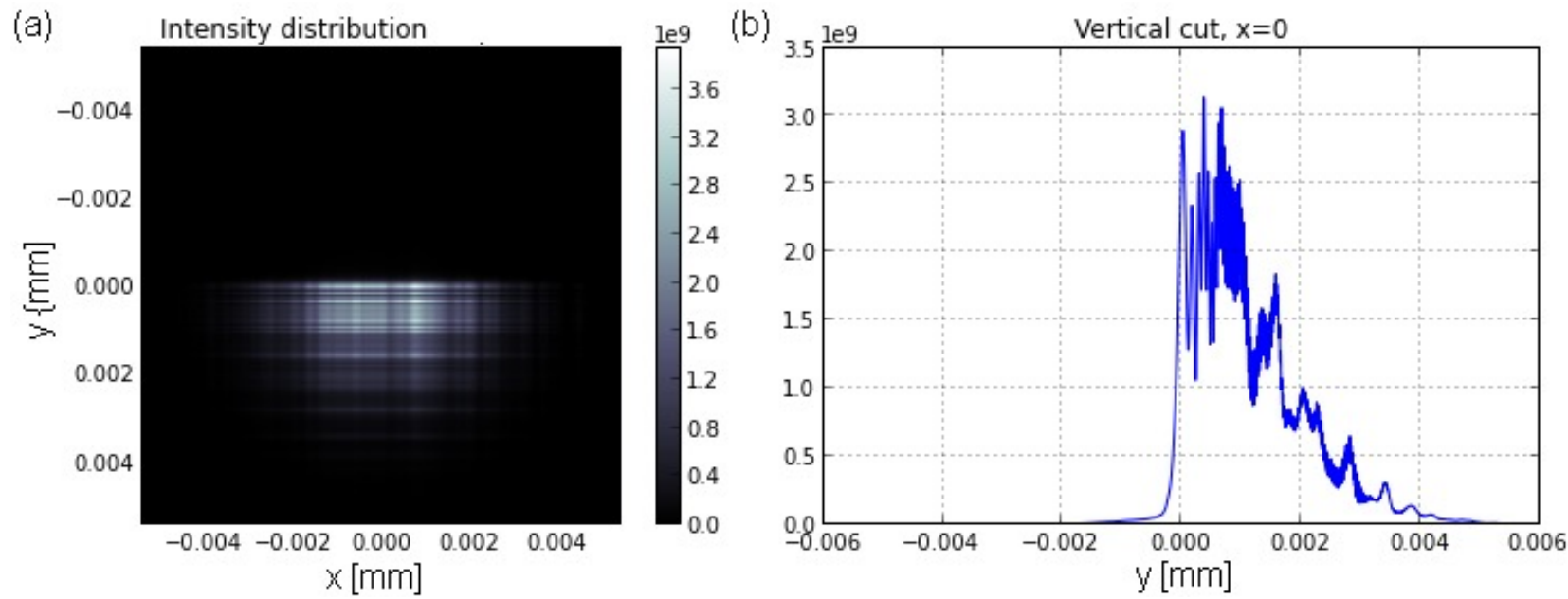

Fig. 7: (a) The profile of the reflected beam $130 \mathrm{~m}$ from the beam-splitter. (b) Vertical cut through the beam-profile at $\mathrm{x}=0 \mathrm{~mm}$.

Figure 8 shows the results of a similar simulation with the same photon energy and distance between the beam-splitter and the experiment, but with an improved surface of the mirror. Here the height error ( peak-to-valley) is only $3 \mathrm{~nm}$. It is obvious that the quality of the reflected beam is improved drastically compared to the beam-profile shown in Fig. 7. The visibility of the first fringe at the cutting edge is $\mathrm{V}=0.17$ while the other fringes only yield visibilities of $\mathrm{V}<0.06$.
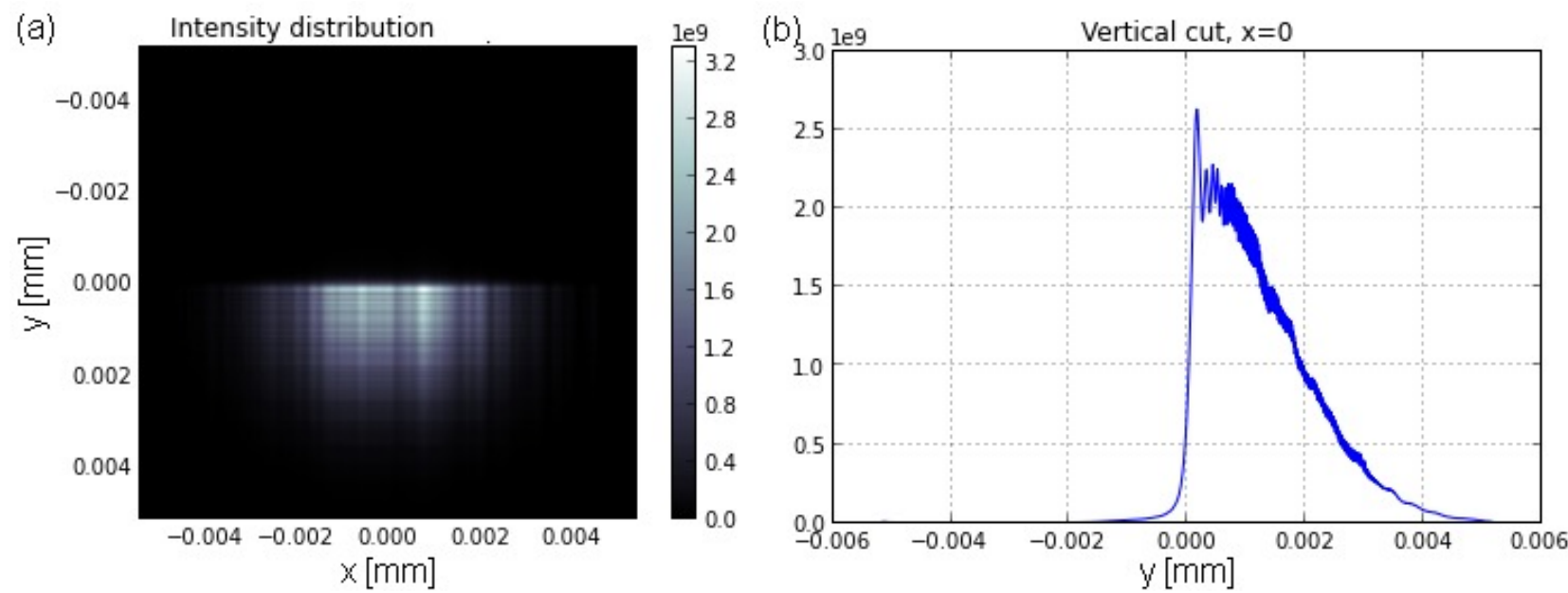

Fig. 8: A similar simulation as shown in Fig. 8, with an improved substrate (height-error $3 \mathrm{~nm}$ peak-to-valley). 


\section{MECHANICAL LAYOUT}

The projected sub-fs resolution as well as the essential pointing stability of the partial beams demand an extensive mechanical stability of the $6 \mathrm{~m}$ long construction. For the SDU at FLASH an intrinsic mechanical stabilization of the entire system is achieved by increasing the stiffness of the whole system. Thereby vibrations are significantly reduced. To ensure the mechanical sturdyness all components are mounted inside an optical bench which consists of an octagonal structure of stainless steel. For the SDU for the European XFEL a similar octagonal structure will be utilized, see Fig. 9.

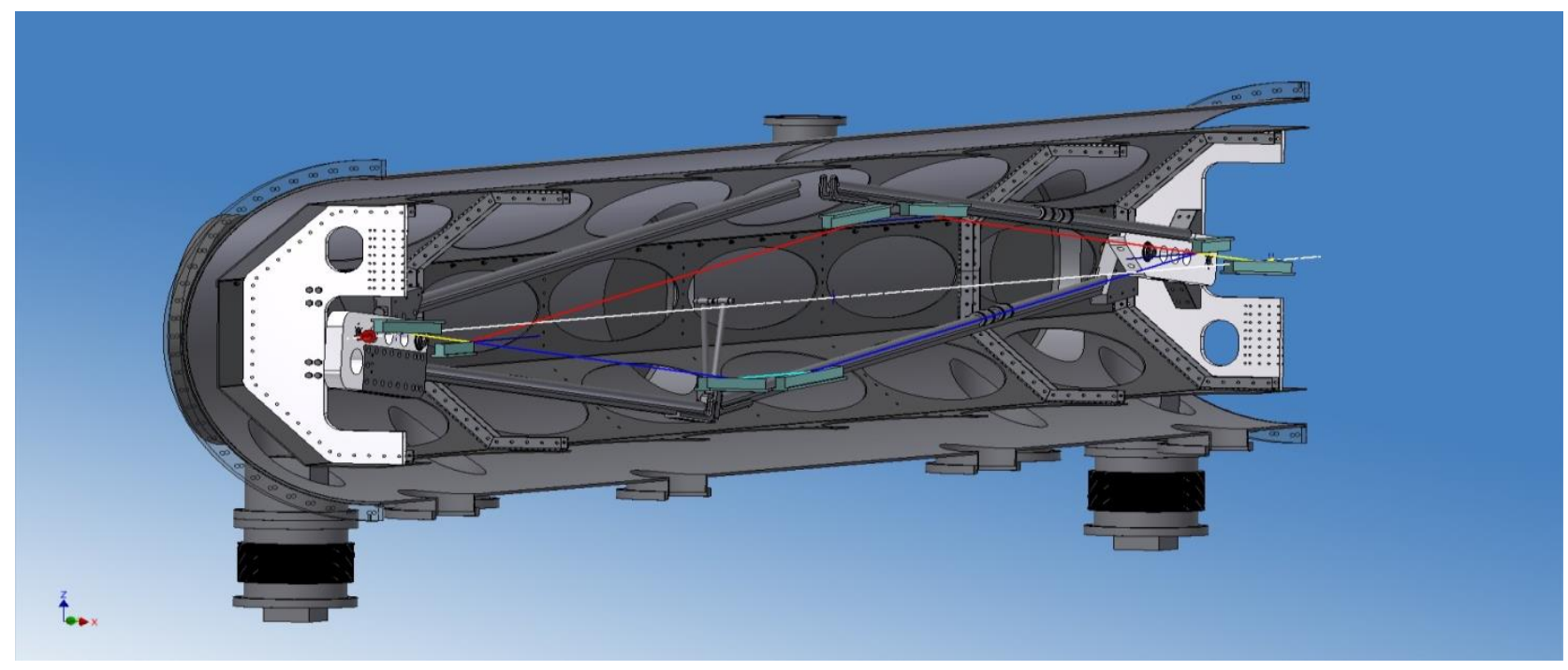

Fig. 9: The mechanical layout of the optical bench with the opto-mechanical components.

The mechanical stability of the optical bench is further improved by supporting frames. As discussed before the FEL beam is divided geometrically and both partial beams travel along two paths whose lengths can be adjusted. The pathlength difference of one beam with respect to the other and in consequence the temporal delay is changed by moving the mirrors of both arms along the $2.4 \mathrm{~m}$ long guide rails. In order to adjust the correct angles for different photon energies all mirrors are turnable and the angle of the guide rails is variable. By moving the mirror the path length which the beam travels along the hypotenuse instead of the (shorter) adjacent of a triangle is varyied, compare Fig. 3. To obtain the designed sub-100 attosecond resolution of the delay this longitudinal motion of the mirrors has to be very precise. Under an grazing angle of $\theta=0.57^{\circ}$ (for $\mathrm{h} \nu=20 \mathrm{keV}$ ) a movement of the mirror of $\Delta \mathrm{l}=10 \mu \mathrm{m}$ results in a pathlength difference of the light of $1.9 \mathrm{~nm}$ which corresponds to a temporal delay of $\Delta \mathrm{t}=6$ as. If the grazing angle is $\theta=2.28^{\circ}$ (for $\mathrm{h} v=5 \mathrm{keV}$ ) the corresponding delay for a movement of $\Delta \mathrm{l}=10 \mu \mathrm{m}$ is $\Delta \mathrm{t}=100$ as. Since the grazing angles of multilayer mirrors depend on the photon energy, an adjustability of the angles of incidence is required. The mirror mountings will therefore posses an angular precission of better than $\Delta \alpha=1 \mu \mathrm{rad}$. For the last mirrors, that reflect the beam to the experiment an additional piezo driven fine tuning is foreseen. In order to provide the experiments with an unaffected XFEL beam the whole optical bench can be moved horizontally so that the beam does not hit any mirror of the SDU. 


\section{CONCLUSION}

A new split- and delay-unit for the European XFEL is designed and constructed for photon energies between $\mathrm{h} v=5 \mathrm{keV}$ and $\mathrm{h} v=20 \mathrm{keV}$. This SDU will serve the users with two time delayed $\mathrm{x}$-ray pulses for $\mathrm{x}$-ray pump / $\mathrm{x}$-ray probe experiments and it will enable a characterization of the temporal properties of the XFEL. Multilayer coated mirrors under gracing incidence will be used in order to provide for a maximum total transmission between $56 \%$ at $\mathrm{hv}=18 \mathrm{keV}$ and $48 \%$ at $h v=8 \mathrm{keV}$, respectively $26 \%$ at $\mathrm{h} v=5 \mathrm{keV}$.

\section{ACKNOWLEDGEMENT}

This work is supported by the BMBF (project: 05K10PM2).

\section{REFERENCES}

[1] M. Altarelli et al., The European X-Ray Free Electron Laser Technical Design Report, DESY Hamburg (2006)

[2] S. Roling et al., Proc. SPIE 8504, 850407 (2012)

[3] C.M. Günther et al., Nat. Phot 5, 99-102 (2011).

[4] R. Mitzner et al., Opt. Expr. 16, 19909-19919 (2008)

[5] P. Emma et al., Nat. Phot. 4, 641-647 (2010)

[6] D. Pile, Nat. Phot. 5, 456-457 (2011)

[7] W. Roseker et al., Opt. Lett. 34, 1768-1770 (2009)

[8] S. Roling et al., Phys. Rev. ST Accel. Beams 14, 080701 (2011)

[9] R. Mitzner et al., Phys. Rev. A 80, 025402 (2009)

[10] M. Krikunova et al., J. Phys. B: At. Mol. Opt. Phys. 45, 105101 (2012)

[11] F. Siewert et al., Nucl. Instrum. Methods Phys. Res. A 635, S52-S57 (2011) 